\title{
Human lung-derived mesenchymal stem cell-conditioned medium exerts in vitro antitumor effects in malignant pleural mesothelioma cell lines
}

\author{
Lourdes Cortes-Dericks", Laurene Froment, Gregor Kocher and Ralph A. Schmid
}

\begin{abstract}
Background: The soluble factors secreted by mesenchymal stem cells are thought to either support or inhibit tumor growth. Herein, we investigated whether the human lung-derived mesenchymal stem cell-conditioned medium (hIMSC-CM) exerts antitumor activity in malignant pleural mesothelioma cell lines H28, H2052 and Meso4.

Methods: hIMSC-CM was collected from the human lung-derived mesenchymal stem cells. Inhibition of tumor cell growth was based on the reduction of cell viability and inhibition of cell proliferation using the XTT and BrdU assays, respectively. Elimination of tumor spheroids was assessed by the anchorage-independent sphere formation assay. The cytokine profile of hIMSC-CM was determined by a chemiluminescence-based cytokine array.

Results: Our data showed that hIMSC-CM contains a broad range of soluble factors which include: cytokines, chemokines, hormones, growth and angiogenic factors, matrix metalloproteinases, metalloproteinase inhibitors and cell-cell mediator proteins. The 48- and 72-hour hIMSC-CM treatments of H28, H2052 and Meso4 cell lines elicited significant decreases in cell viability and inhibited cell proliferation. The 72-hour hIMSC-CM incubation of H28 cells completely eliminated the drug-resistant sphere-forming cells, which is more potent than twice the half maximal inhibitory concentration of cisplatin.

Conclusions: Our findings indicate that the cell-free hIMSC-CM confers in vitro antitumor activities via soluble factors in the tested mesothelioma cells and, hence, may serve as a therapeutic tool to augment the current treatment strategies in malignant pleural mesothelioma.
\end{abstract}

Keywords: Malignant pleural mesothelioma, Human lung mesenchymal stem cell-conditioned medium, Soluble factors, Sphere-forming cells, Cisplatin

\section{Introduction}

In contrast to the early paradigm of cell replacement and differentiation of mesenchymal stem cells (MSCs) as a therapeutic mechanism, reports are accumulating that cell secretions are responsible for their beneficial effects [1]. MSCs are known to secrete a wide range of bioactive molecules such as growth factors, cytokines and chemokines that regulate their biology in an autocrine or paracrine manner in accordance with the environmental niche [2]. It has been recognized that MSC activities are

\footnotetext{
* Correspondence: cortes-dericks@gmx.de

University Hospital Berne, Department of Clinical Research, Division of General Thoracic Surgery, Berne, Switzerland
}

mediated by secreted biomolecules, highlighting the importance of using MSC-derived conditioned media (MSC-CM) in regenerative medicine as well as in cancer therapy. Clinically, the use of cell-free MSC-CM may represent a better therapeutic tool compared with stem cell-based therapy as the former is easier to prepare, maintain, and transport to appropriate sites, and may also have less complications related to issues on cell transplants [3].

When MSCs arrive at a tumor niche, they produce a variety of soluble factors that either positively or negatively influence tumor growth [4]. Several reports have accounted for the antitumorigenic capacity of MSCs. 
Khakoo et al. [5] have demonstrated that intravenously injected human MSCs (hMSCs) in a mouse model of sarcoma could potently inhibit tumor growth. Cell lysates or supernatants of Wharton's jelly-derived MSCs have manifested a capacity to inhibit cell growth of breast cancer, ovarian tumor and osteosarcoma cell lines, indicating tumor inhibitory properties [6]. Although hMSCs have been demonstrated to suppress proliferation and induce apoptosis of SKMES-1 and A549 lung adenocarcinoma cells via some soluble factors [7], no current knowledge of hMSC-CM actions on malignant pleural mesothelioma (MPM) is known.

MPM is a highly aggressive, chemoresistant lung cancer with a median survival of $<1$ year after diagnosis $[8,9]$. Thus, new therapeutic strategies are imperative to improve patient survival. Given that soluble factors secreted by MSCs are supposed to mediate beneficial effects, we investigated whether the cell-free hIMSC-CM exerts an antitumor capacity in three MPM cell lines.

\section{Materials and methods Cell culture}

The H28 and H2052 mesothelioma cell lines (LCD Promochem, France) were cultured in RPMI 1640 (PAA, Austria) containing $10 \%$ fetal bovine serum (FBS; PAA, Austria) and $1 \%$ penicillin/streptomycin (Invitrogen, Switzerland). ACC-Meso-4 cell line was obtained from Riken Cell Bank, Resource No: RBRC-RCB2293 (Ibaraki, Japan) and cultured using the above-mentioned culture medium. Cells were maintained at $37^{\circ} \mathrm{C}, 95 \%$ humidity and $5 \% \mathrm{CO}_{2}$.

\section{Isolation and characterization of hIMSCs}

We have previously identified human lung parenchyma mesenchymal stromal cells, which are referred as human lung-derived mesenchymal stem cells (hIMSCs) in this study. hlMSCs were isolated by using our established protocol as reported in our previous work [10], which includes immunophenotyping and in vitro mesenchymal trilineage differentiation into chondrocytes, adipocytes and osteocytes (see Additional file 1).

\section{Collection of hIMSC-CM}

Conditioned medium $(\mathrm{CM})$ was collected from hlMSCs at passage 3. Cells were cultured at a density of $5 \times 10^{4}$ in $10 \mathrm{~cm}$ cell culture dish containing MCDB 201 supplemented with ITS, $1 \%$ antibiotic/antimytotic reagent (Hyclone), $1 \% \mathrm{FBS}$ and $20 \mathrm{ng} / \mu \mathrm{l}$ endothelial growth factor at $37{ }^{\circ} \mathrm{C}$ in a humid atmosphere with $5 \% \mathrm{CO}_{2}$. Upon reaching 70-80\% confluency, cells were washed once with phosphate-buffered saline (PBS) and then re-incubated with a serum-free MCDB 201 medium for 24 hours. The CM (supernatant) was then collected and centrifuged at $1200 \times \mathrm{g}$ for 10 minutes to remove cell components and was sterilized by passing through a $0.2-\mu \mathrm{m}$ cell filter (BD Biosciences, Switzerland). Aliquots were subjected to cytokine array analysis or frozen at $-80{ }^{\circ} \mathrm{C}$ for future applications. hlMSC-CM was used as it was, without dilution or addition of serum in all of the experiments in this project.

\section{Cell proliferation and cell viability assays}

The XTT and BrdU assays were performed to assess the effect of hlMSC-CM on cell viability and cell proliferation, respectively (see Additional file 2).

\section{Cytokine array assay}

The cytokine profile of hlMSC-CM was determined using the Human Angiogenesis Antibody Array (see Additional file 3).

\section{Sphere formation}

The sphere formation assay used to evaluate the formation of nonadherent multicellular spheroids is described in Additional file 4.

\section{Drug treatment}

For drug treatment, cisplatin (diamminedichloroplatinum (II); Bristol Myers Squibb, Switzerland) was used. Cells were seeded at a density of $5 \times 10^{4}$ cells/well in six-well culture dishes and incubated for 24 hours prior to 48- and 72-hour treatments with the half maximal inhibitory concentration $\left(\mathrm{IC}_{50}\right)$ or twice the $\mathrm{IC}_{50}$ of cisplatin (twice the $\mathrm{IC}_{50}$ means $2 \times$ the concentration of the $\mathrm{IC}_{50}$ value). The specified concentration corresponds to the previously determined $\mathrm{IC}_{50}$ value of the three MPM cell lines: H28 $(15 \mu \mathrm{M}), \mathrm{H} 2052(13 \mu \mathrm{M})$, and Meso4 $(12 \mu \mathrm{M})$ [11]. Following the cisplatin treatments at $37{ }^{\circ} \mathrm{C}$, cells were washed with PBS, trypsinized, and single-cell suspension was prepared for sphere formation assay.

\section{Statistical analysis}

Data are reported as means \pm standard deviations. For analysis of differences in multiple groups, one-way analysis of variance and Bonferroni's post-hoc test were performed using Prism 6.0 (GraphPad Software, USA). A $p$ value $<0.05$ was considered significant.

\section{Results}

\section{hIMSC-CM contains soluble factors}

We have previously identified hlMSCs exhibiting plastic adherence, the immunophenotype and trilineage differentiation capacity consistent with the established features of MSCs [10, 12, 13]. We initially investigated whether our hlMSC-CM contains soluble factors. We therefore collected the CM of the hlMSCs grown for 24 hours in the absence of FBS and analyzed it using the 
cytokine array. hlMSC-CM contained a broad range of soluble factors which included: cytokines, chemokines, hormones, growth factors, neurotrophic factors, endocrine and angiogenic factors, matrix metalloproteinases (MMPs), metalloproteinase inhibitors (TIMPs) and cellcell mediator proteins (Fig. 1).

\section{hIMSC-CM inhibits cell proliferation and reduces cell viability in three MPM cells lines}

We studied the effect of hlMSC-CM on the proliferative activity of $\mathrm{H} 28, \mathrm{H} 2052$ and Meso4 using the BrdU assay. The 48- and 72-hour treatments with hlMSC-CM elicited significant reductions in cell proliferation of $\mathrm{H} 28$ (48 hours $-74 \%$; 72 hours $-76 \%$ ), H2052 (48 hours $-62 \% ; 72$ hours $-64 \%$ ) and Meso4 (48 hours $-35 \% ; 72$ hours $-55 \%$ ) relative to the nontreated cells (Fig. 2a-c). We also investigated the effect of hlMSC-CM on cell viability after the treatment periods of 48 and 72 hours using the XTT assay. hlMSC-CM evoked significant reductions in cell viability in all tested cell lines: H28 (48 hours $-69 \%$; 72 hours $-81 \%$ ), H2052 (48 hours $-25 \%$; 72 hours $25.3 \%$ ), Meso4 (48 hours $-26.3 \%$; 72 hours $-31 \%$ ) compared with the nontreated cells (Fig. $2 \mathrm{~d}-\mathrm{f}$ ).

\section{hIMSC-CM eliminates sphere-forming phenotype in H28 cells}

In our previous work, we found cisplatin-resistant tumor spheres in H28, H2052 and Meso4 indicating the presence of putative cancer stem cells (CSCs), which may, in part, be responsible for drug resistance [11]. Hence, we investigated the ability of hlMSC-CM to eliminate these cells, and also compared its efficacy with cisplatin, a standard chemotherapy in the treatment of MPM [8, 9]. hlMSC-CM significantly reduced the sphere-forming efficiency by $70 \%$ in $\mathrm{H} 28$ cells after 48 hours and, unexpectedly, fully eliminated them after the 72-hour treatment (Fig. 3a). These effects were not observed in H2052 and Meso4 cells (Fig. $3 \mathrm{~b}$ and c). Representative images of the nontreated and hIMSC-CM-treated MPM cells are shown in Fig. 3d-f.

We then compared the inhibitory effect of hlMSC$\mathrm{CM}$ on the growth of tumor spheres with that of $\mathrm{IC}_{50}$ and twice the $\mathrm{IC}_{50}$ of cisplatin in $\mathrm{H} 28$ cells. The 48-hour hlMSC-CM treatment significantly reduced the sphere-forming efficiency by $74 \%$, whereas the 72-hour incubation completely eliminated the tumor spheres (Fig. 4a). Using the $\mathrm{IC}_{50}$ of cisplatin, we obtained significant $50 \%$ reductions in sphere efficiency after the 48- and 72-hour treatments (Fig. 4b), and significant decreases of 68 and $73 \%$ after analogous incubation periods using twice the $\mathrm{IC}_{50}$ of cisplatin (Fig. 4c). Our results showed that hIMSC-CM is capable of fully eliminating the sphere-forming phenotype in $\mathrm{H} 28$ cells, and that this effect has a higher efficacy compared with twice the $\mathrm{IC}_{50}$ of cisplatin. Representative images of the nontreated and hlMSC-CM-treated H28 cells are shown in Fig. 4d-f.

\section{Discussion}

One beneficial effect of MSCs is their inherent antitumor capacity-a current topic of investigation because of its future role in cancer therapy. Here, we report that hlMSC-CM contains a broad range of soluble factors, which conferred inhibition of cell proliferation and a decrease in cell viability in three MPM cell lines. Importantly, hlMSC-CM completely eliminated the cisplatin-resistant, sphere-forming cells.

We found a huge panel of cytokines, chemokines, growth, neurotrophic, endocrine and angiogenic factors, MMPs, TIMPs and cell-cell mediator proteins in our hIMSC-CM that elicited suppression of cell growth and reduced cell viability in the tested mesothelioma cells. These data substantiate the current paradigm that MSC-dependent beneficial effects are mediated primarily by secreted factors [1]. Evidences for the soluble factor-mediated antitumor capacity of hMSC-CM have been reported such as the reduction of SK-MES-1 and A549 lung cancer cell proliferation by a downregulation of vascular endothelial growth factor (VEGF) expression and by an increased apoptosis ratio compared with the non-hMSC-CM-treated cells [7]. The in vitro treatment of ovarian cancer cells with heat shocked adipose stem cell-derived CM and amniotic-derived stem cells have resulted in decreased tumor cell viability compared with controls by inducing greater nuclear condensation and growth cycle arrest of tumor cells. Cytokine array of the CM demonstrated that the observed antitumor effect was mediated by angiogenin, IGF-binding protein 4, NT-3 and chemokine ligand 18 [14]. In a coculture study of lung adenocarcinoma (LAC) cell lines and MSCs or MSC-CM, Wang et al. [15] have shown evidence that oncostatin $\mathrm{M}$, a differentiation-promoting cytokine, mediated the MSC-dependent inhibition of tumorigenicity and activation of mesenchymal epithelial transition in LAC cells. Extracellular matrix components produced by human umbilical cord blood MSCs have also contributed to the growth arrest of metastatic cancer cells by an upregulation of tumor suppressor phosphatase and tensin homolog (PTEN) in the tumor cells [16]. The data in our cytokine array include factors that are likely involved in conferring antitumorigenic effects of MSCs such as angiogenin, VEGF, transforming growth factor-beta (TGF-b) and hepatocyte growth factor (HGF) [14, 17]. They also showed increased levels of biomolecules that have been linked to 


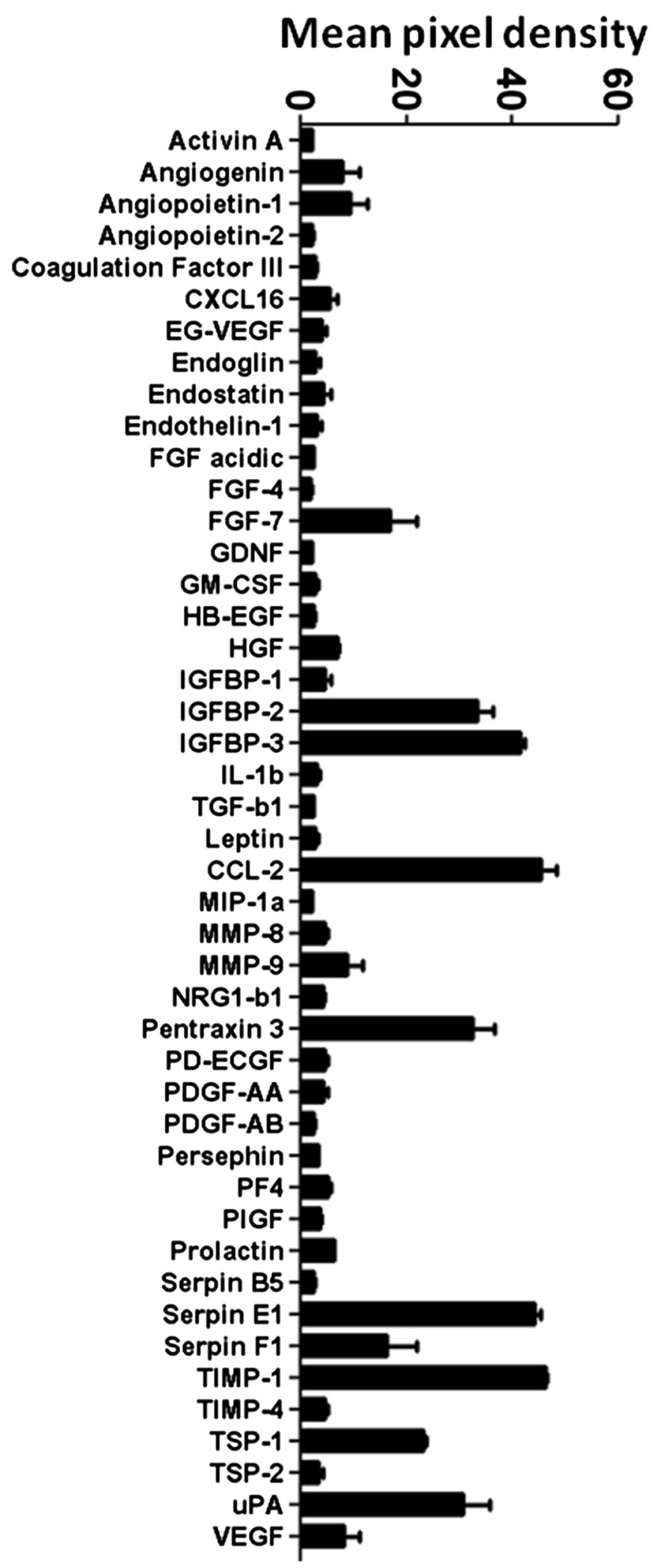

Fig. 1 hIMSC-CM contains a broad range of soluble factors. The CM (supernatant) of hIMSCs grown for 24 hours in a culture medium without FBS was collected and subjected to a cytokine array assay as described in the Materials and methods. Results are representative of one of the three independent experiments 


\section{$\mathrm{H} 28$}
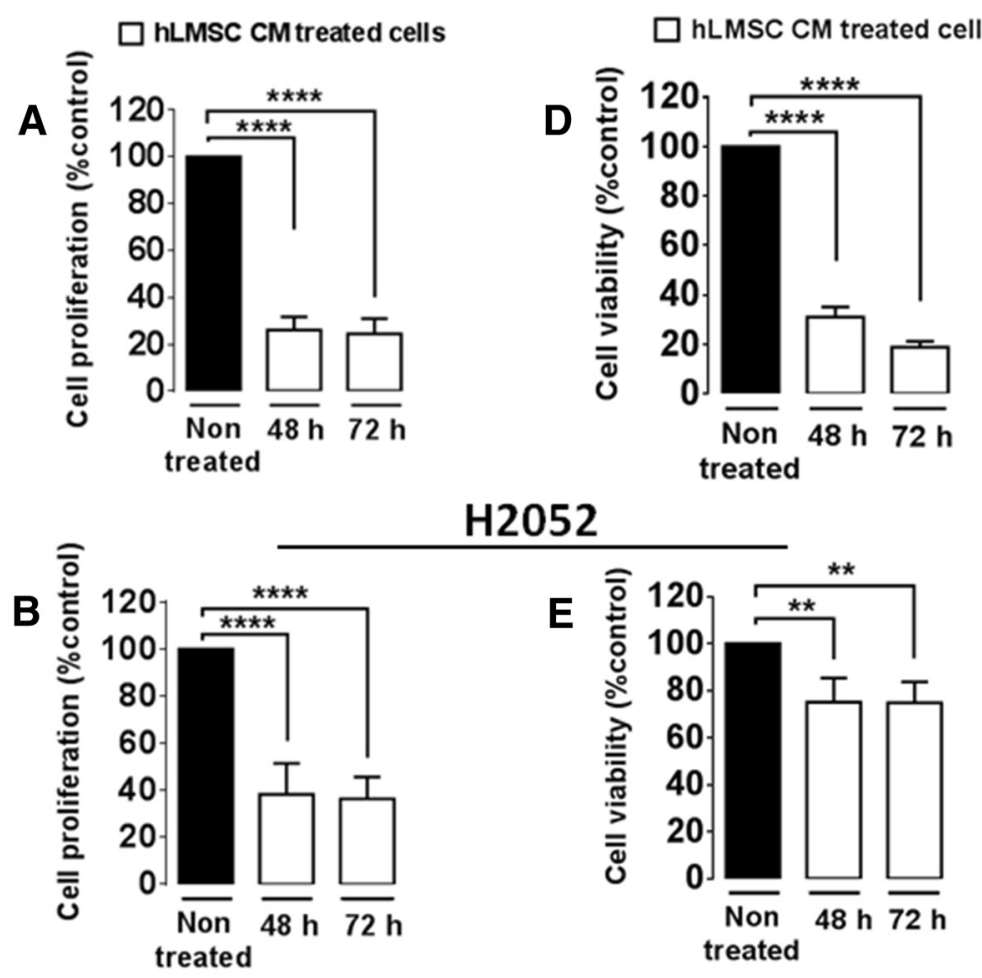

Meso4

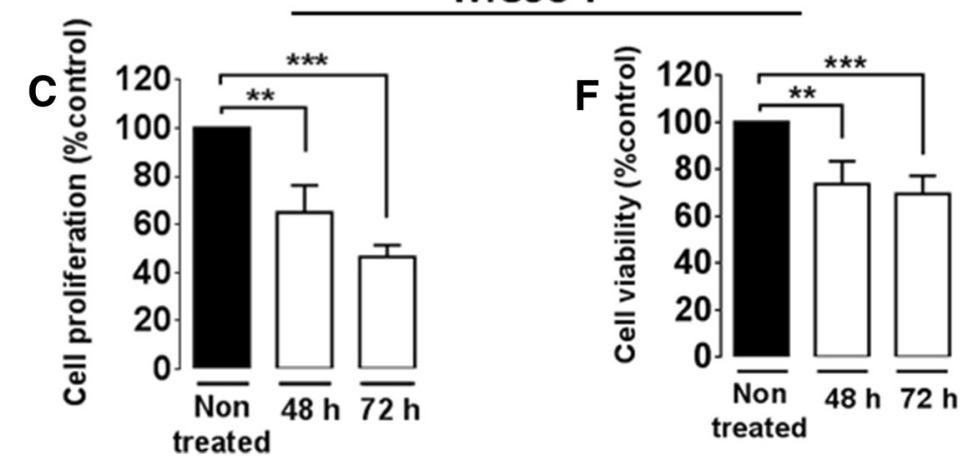

Fig. 2 The inhibitory effect of hIMSC-CM on cell proliferation and reduction of cell viability in the three MPM cells lines. Significant inhibitions on cell proliferation of hIMSC-CM-treated H28 (a), H2052 (b) and Meso4 (c) cells were expressed as the percentage of proliferation relative to the nontreated (control) cells as determined by the BrdU assay. Reductions on cell viability in hIMSC-CM-treated H28 (d), H2052 (e) and Meso4 (f) cells were expressed as a percentage of cell viability relative to nontreated cells as evaluated by the XTT assay. Results are the means \pm standard deviations of three independent experiments each. ${ }^{* *} p<0.01,{ }^{* * *} p<0.001,{ }^{* * *} p<0.0001$. $h$ Hours, hLMSC CM Human lung-derived mesenchymal stem cell-conditioned media

diverse roles in the malignant setting, which include IGFBP-1, IGFBP-2, CCL2, Pentraxin, SerpinE1, SerpinE2, TIMP-1 and uPA [18-23]. Further investigation is necessary to identify the specific factors that are implicated in the observed antitumorigenic property of hlMSC-CM in the tested mesothelioma cells.

MSCs have diverse effects on tumor growth. In contrast to the reported tumor-promoting capacity of MSCs, our findings showed a tumor-inhibiting effect of hlMSC-CM in the tested MPM cell lines. Tumor-promoting activity is exerted either by paracrine secretion of growth factors and antiapoptotic factors, or by differentiating into tumor-associated fibroblasts, which can enhance tumor growth, metastasis formation and therapy resistance [4]. Adversely, MSCs and/or MSC-derived $\mathrm{CM}$ also confer antitumorigenic responses as have 


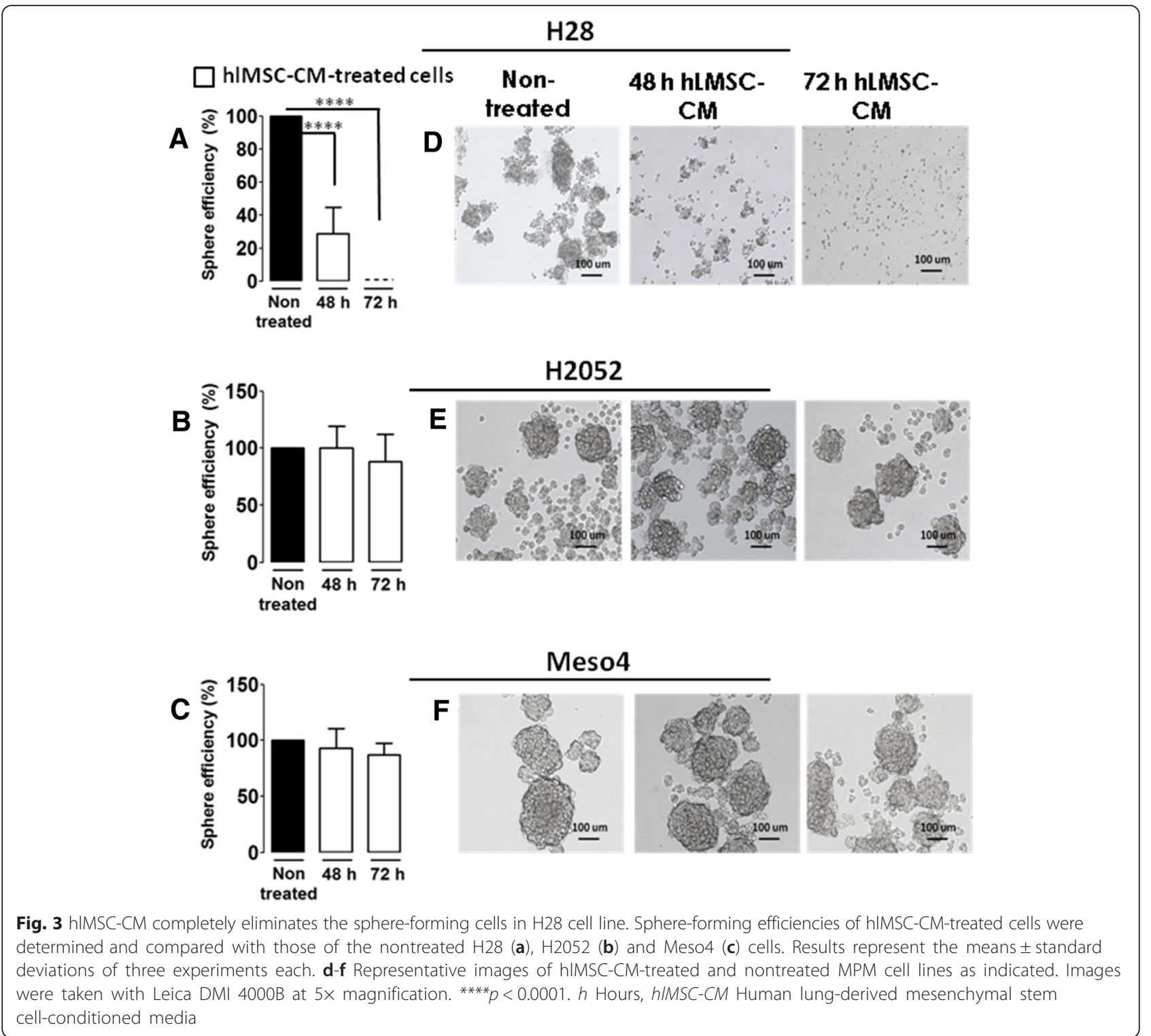

been demonstrated by the inhibition of tumor growth in leukemia [24], prostate carcinoma [25], Kaposi's sarcoma [5], colon carcinoma [26], breast cancer [27] and murine lung cancer [28] under in vitro and in vivo conditions. Diverse mechanisms have been reported to be involved in the antitumor potential of MSCs that include apoptosis caused by an upregulation of TRAIL, cell cycle arrest, blocking of the PI3K/AkT pathway, expression of tumor suppressor genes, downregulation of the Wnt pathway, expression of DKK1 and cytokinemediated process. Operationally, MSCs have also been proven to home to tumor sites and exert suppression of tumor growth amongst others [29]. Our data point to a soluble factor-mediated antitumor effect of hIMSC-CM reflecting the notion that hMSCs have the innate capacity to produce biomolecules in response to the tumor microenvironment.

Sphere formation represents one of the basic features of CSCs, a rare subpopulation within a tumor, which is supposed to be a crucial player in the initiation, invasiveness and drug tolerance in different tumors including MPM [11, 30-35]. A striking property of hLMSC-CM observed in this study is its capacity to fully eliminate the sphere-forming phenotype in H28 cells. The failure of hlMSC-CM to abolish the sphere-forming cells in H2052 and Meso4 may be due to the differences in the histological composition of the tested cell lines. MPM is divided into three histological subtypes: epitheloid, sarcomatoid and biphasic [9]. Multicellular spheroids possess heterogeneity similar to that of tumor in vivo 


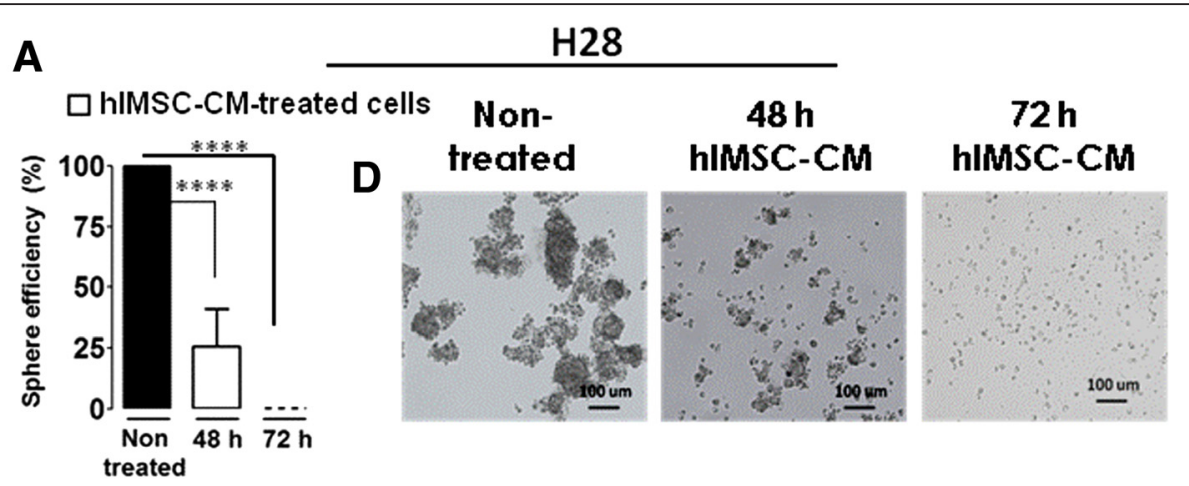

$\mathrm{H} 28$

B

B $\square \mathrm{IC} \mathrm{C}_{50}$ cisplatin-treated cells Non-

$48 \mathrm{~h}$

$72 \mathrm{~h}$

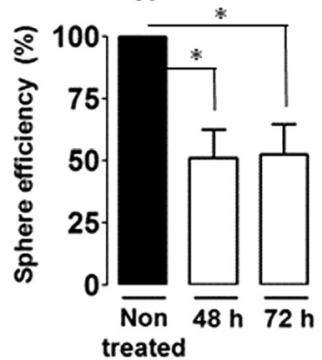

E treated

cisplatin, $I C_{50}$ cisplatin, $I C_{50}$
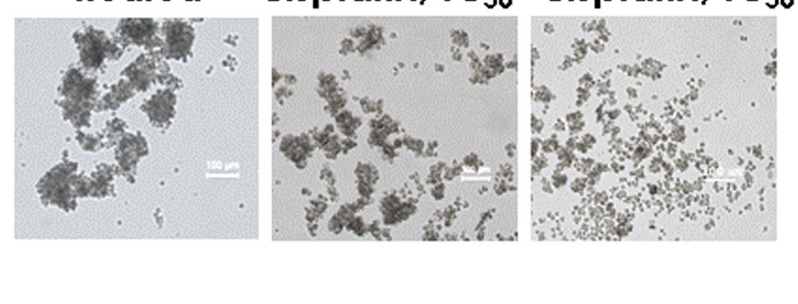

H28

$C_{\square 2 \times 1 C_{50} \text { cisplatin-treated cells }}$
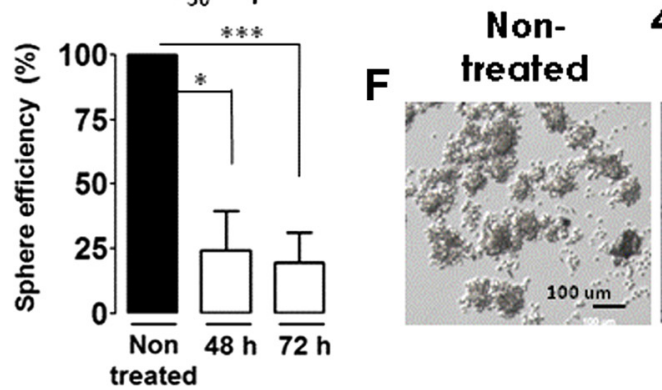

$48 \mathrm{~h}$ cisplatin, $72 \mathrm{~h}$ cisplatin, $2 \times I C_{50}$ $2 \times I_{50}$
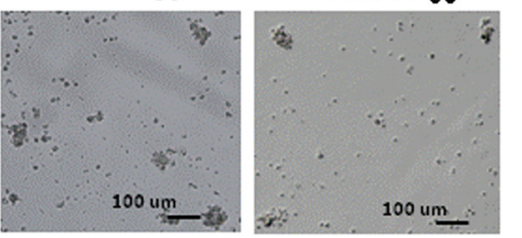

Fig. 4 hIMSC-CM shows higher efficacy than twice the $\mathrm{IC}_{50}$ of cisplatin in inhibiting the growth of tumor spheres in $\mathrm{H} 28$ cells. $\mathrm{H} 28$ cells were treated in the absence or presence hIMSC-CM (a), $1 \times I C_{50}$ cisplatin (b) and $2 \times I C_{50}$ cisplatin (c) at the indicated periods after which sphereformation assays were performed. Results indicate the sphere efficiencies of the hIMSC-CM-and cisplatin-treated cells relative to the nontreated controls. d-f Representative images of the hIMSC-CM-, cisplatin-treated cells and nontreated cells as indicated. Data represent the means and \pm standard deviations of three independent experiments each. ${ }^{*} p<0.05,{ }^{* * *} p<0.001,{ }^{* * * *} p<0.0001$. $h$ Hours, hIMSC-CM Human lung-derived mesenchymal stem cell-conditioned media, $/ C_{50}$ Half maximal inhibitory concentration

and their drug resilience has appeared to be analogous to the natural resistance observed in patient tumors [36]. Therefore, testing for sphere formation after drug treatment would represent a reliable method to evaluate chemoresistance, not only in vitro but also in an in vivo setting. Considering this, hlMSC-CM, which showed a high potency to eliminate tumor spheres, may be used to resolve drug tolerance in the epitheliod type of MPM as represented by the H28 cell line. Cisplatin is one of the standard components in the treatment of MPM, which as yet results in inadequate patient therapy [8]. Because the efficacy of
hlMSC-CM to eliminate the sphere-forming cells in $\mathrm{H} 28$ is higher compared with twice the $\mathrm{IC}_{50}$ of cisplatin, it stands to reason that combining hlMSC-CM with conventional drugs may improve the current treatment modalities in MPM.

\section{Conclusion}

Our study demonstrates that hlMSC-CM exerts in vitro antitumor effects in three MPM cell lines via soluble factors, which offers a potentially useful tool to augment the standard therapy in MPM. 


\section{Additional files}

Additional file 1: Isolation and characterization of hIMSCs. (DOCX $16 \mathrm{~kb}$ )
Additional file 2: Cell proliferation and cell viability assays. (DOCX $11 \mathrm{~kb}$ )
Additional file 3: Cytokine array assay. (DOCX $10 \mathrm{~kb})$
Additional file 4: Sphere formation. (DOCX $10 \mathrm{~kb})$

\section{Abbreviations}

CM: Conditioned media; CSC: Cancer stem cell; FBS: Fetal bovine serum; hIMSC: Human lung mesenchymal stem cell; hMSC: Human mesenchyma stem cell; $I_{50}$ : Half maximal inhibitory concentration; LAC: Lung adenocarcinoma; MMP: Matrix metalloproteinases; MPM: Malignant pleural mesothelioma; MSC: Mesenchymal stem cell; PBS: Phosphate-buffered saline; TIMP: Metalloproteinase inhibitor; VEGF: Vascular endothelial growth factor.

\section{Competing interests}

The authors declare that they have no competing interests.

\section{Authors' contributions}

LCD conceived and designed the study, analysed the data and wrote the manuscript. LF contributed to the design of the study, performed assays, collected and graphed all experimental data. GK gave suggestions and ensured that important issues were appropriately investigated in the study. RAS gave final approval of the manuscript. All authors read and approved the final manuscript.

Received: 2 September 2015 Revised: 21 December 2015 Accepted: 18 January 2016 Published online: 09 February 2016

\section{References}

1. Madrigal M, Rao KS, Riordan NH. A review of therapeutic effects of mesenchymal stem cell secretions and induction of secretory modification by different culture methods. J Transl Med. 2014;12:260.

2. Chan JKY, Lam PYP. Human mesenchymal stem cells and their paracrine factors for the treatment of brain tumors. Cancer Gene Ther. 2013;20:539-43.

3. Linero I, Chaparro O. Paracrine effect of mesenchymal stem cells derived from human adipose tissue in bone regeneration. PLoS One. 2014;9:e107001.

4. Houthuijzen JM, Daenen LGM, Roodhart JML, Voest EE. The role of mesenchymal stem cells in anti-cancer drug resistance and tumour progression. Br J Cancer. 2012;106:1901-6.

5. Khakoo AY, Pati S, Anderson SA, Reid W, Elshal MF, Rovira II, et al. Human mesenchymal stem cells exert potent antitumorigenic effects in a model of Kaposi's sarcoma. J Exp Med. 2006:203:1235-47.

6. Gauthaman K, Yee FC, Cheyyatraivendran S, Biswas A, Choolani M, Bongso A. Human umbilical cord Wharton's jelly stem cell (hWJSC) extracts inhibit cancer cell growth in vitro. J Cell Biochem. 2012;113:2027-39.

7. Li L, Tian H, Chen Z, Yue W, Li S, Li W. Inhibition of lung cancer cell proliferation mediated by human mesenchymal stem cells. Acta Biochim Biophys Sin. 2011;43:143-8.

8. Opitz I. Management of malignant pleural mesothelioma-The European experience. J Thorac Dis. 2014;6 Suppl 2:S238-52.

9. Porpodis K, Zarogoulidis P, Boutsikou E, Papaioannou A, Machairiotis N, et al. Malignant pleural mesothelioma: current and future perspectives. J Thorac Dis. 2013;5(Suppl 4):S397-406

10. Karoubi G, Cortes-Dericks L, Breyer I, Schmid RA, Dutly AE. Identification of mesenchymal stromal cells in human lung parenchyma capable of differentiating into aquaporin 5-expressing cells. Lab Investig J Tech Methods Pathol. 2009:89:1100-14.

11. Cortes-Dericks L, Froment L, Boesch R, Schmid RA, Karoubi G. Cisplatinresistant cells in malignant pleural mesothelioma cell lines show ALDH(high)CD44(+) phenotype and sphere-forming capacity. BMC Cancer. 2014;14:304

12. Dominici M, Le Blanc K, Mueller I, Slaper-Cortenbach I, Marini F, Krause D, et al. Minimal criteria for defining multipotent mesenchymal stromal cells. The International Society for Cellular Therapy position statement. Cytotherapy. 2006;8:315-7.

13. Nombela-Arrieta C, Ritz J, Silberstein LE. The elusive nature and function of mesenchymal stem cells. Nat Rev Mol Cell Biol. 2011;12:126-31.
14. Cho JA, Park H, Kim HK, Lim EH, Seo SW, Choi JS, et al. Hyperthermiatreated mesenchymal stem cells exert antitumor effects on human carcinoma cell line. Cancer. 2009;115:311-23.

15. Wang M-L, Pan C-M, Chiou S-H, Chen W-H, Chang H-Y, Lee OK-S, et al. Oncostatin M modulates the mesenchymal-epithelial transition of lung adenocarcinoma cells by a mesenchymal stem cell-mediated paracrine effect. Cancer Res. 2012;72:6051-64.

16. Sun B, Yu K-R, Bhandari DR, Jung J-W, Kang S-K, Kang K-S. Human umbilical cord blood mesenchymal stem cell-derived extracellular matrix prohibits metastatic cancer cell MDA-MB-231 proliferation. Cancer Lett. 2010;296:178-85.

17. Baraniak PR, McDevitt TC. Stem cell paracrine actions and tissue regeneration. Regen Med. 2010;5:121-43.

18. Martin JL, Coverley JA, Pattison ST, Baxter RC. Insulin-like growth factorbinding protein-3 production by MCF-7 breast cancer cells: stimulation by retinoic acid and cyclic adenosine monophosphate and differential effects of estradiol. Endocrinology. 1995;136:1219-26.

19. Li M, Knight DA, Snyder LA, Smyth MJ, Stewart TJ. A role for CCL2 in both tumor progression and immunosurveillance. Oncolmmunology. 2013;2, e25474.

20. Kondo S, Ueno H, Hosoi H, Hashimoto J, Morizane C, Koizumi F, et al. Clinical impact of pentraxin family expression on prognosis of pancreatic carcinoma. Br J Cancer. 2013;109:739-46.

21. Fortenberry $Y$. The role of serpins in tumor cell migration. Biol Chem. 2015; 396:205-13.

22. Bloomston M, Shafii A, Zervos EE, Rosemurgy AS. TIMP-1 overexpression in pancreatic cancer attenuates tumor growth, decreases implantation and metastasis, and inhibits angiogenesis. J Surg Res. 2002;102:39-44.

23. Dass K, Ahmad A, Azmi AS, Sarkar SH, Sarkar FH. Evolving role of uPA/UPAR system in human cancers. Cancer Treat Rev. 2008:34:122-36.

24. Ramasamy R, Lam EW-F, Soeiro I, Tisato V, Bonnet D, Dazzi F. Mesenchymal stem cells inhibit proliferation and apoptosis of tumor cells: impact on in vivo tumor growth. Leukemia. 2007;21:304-10.

25. Chanda D, Isayeva T, Kumar S, Hensel JA, Sawant A, Ramaswamy G, et al. Therapeutic potential of adult bone marrow-derived mesenchymal stem cells in prostate cancer bone metastasis. Clin Cancer Res Off J Am Assoc Cancer Res. 2009:15:7175-85.

26. Ohlsson LB, Varas L, Kjellman C, Edvardsen K, Lindvall M. Mesenchymal progenitor cell-mediated inhibition of tumor growth in vivo and in vitro in gelatin matrix. Exp Mol Pathol. 2003;75:248-55.

27. Ayuzawa R, Doi C, Rachakatla RS, Pyle MM, Maurya DK, Troyer D, et al. Naïve human umbilical cord matrix derived stem cells significantly attenuate growth of human breast cancer cells in vitro and in vivo. Cancer Lett. 2009:280:31-7.

28. Maestroni GJ, Hertens E, Galli P. Factor(s) from nonmacrophage bone marrow stromal cells inhibit Lewis lung carcinoma and B16 melanoma growth in mice. Cell Mol Life Sci CMLS. 1999;55:663-7.

29. Ramdasi S, Sarang S, Viswanathan C. Potential of mesenchymal stem cell based application in cancer. Int J Hematol-Oncol Stem Cell Res. 2015;9:95-103.

30. Levina V, Marrangoni AM, DeMarco R, Gorelik E, Lokshin AE. Drug-selected human lung cancer stem cells: cytokine network, tumorigenic and metastatic properties. PLoS One. 2008:3:e3077.

31. Liu J, Ma L, Xu J, Liu C, Zhang J, Liu J, et al. Spheroid body-forming cells in the human gastric cancer cell line MKN-45 possess cancer stem cell properties. Int J Oncol. 2013;42:453-9.

32. Liu W-D, Zhang T, Wang C-L, Meng H-M, Song Y-W, Zhao Z, et al. Sphereforming tumor cells possess stem-like properties in human fibrosarcoma primary tumors and cell lines. Oncol Lett. 2012;4:1315-20.

33. Saleem S, Jamshed A, Faisal S, Hussain R, Tahseen M, Loya A, et al. Patterns of cancer cell sphere formation in primary cultures of human oral tongue squamous cell carcinoma and neck nodes. Cancer Cell Int. 2014;14:542.

34. Zhang G, Xiong $\mathrm{K}, \mathrm{Ma}$ W, Xu W, Zeng $\mathrm{H}$. Initiate tumors with single cell spheres formed in serum-containing medium. J Cancer. 2015:6:901-12.

35. Sun F-F, Hu Y-H, Xiong L-P, Tu X-Y, Zhao J-H, Chen S-S, et al. Enhanced expression of stem cell markers and drug resistance in sphere-forming non-small cell lung cancer cells. Int J Clin Exp Pathol. 2015:8:6287-300.

36. Desoize B, Jardillier J-C. Multicellular resistance: another mechanism for multidrug resistance? Crit Rev Oncol Hematol. 2000:36:193-207. 